Témoigner Témoigner. Entre histoire et mémoire

Getuigen Revue pluridisciplinaire de la Fondation Auschwitz

$118 \mid 2014$

Au nom des victimes. Dictature et terreur d'État en Argentine, Chili et Uruguay

\title{
Tragédie vs catastrophe
}

Entretien avec Annette Becker, historienne de la Grande Guerre et des violences extrêmes du XX ${ }^{\mathrm{e} o}$ siècle

Catastrophe vs. Tragedy: Interview with Annette Becker, historian of the Great

War and of extreme violence in the $20^{\text {th }}$ century

Tragedie vs. Catastrofe. Interview met Annette Becker, historica en specialiste op het gebied van de Grote Oorlog en het extreme geweld van de $20^{e}$ eeuw

\section{Annette Becker}

\section{(2) OpenEdition \\ Journals}

Édition électronique

URL : http://journals.openedition.org/temoigner/897

DOI : 10.4000/temoigner.897

ISSN : 2506-6390

Éditeur :

Éditions du Centre d'études et de documentation Mémoire d'Auschwitz, Éditions Kimé

Édition imprimée

Date de publication : 1 octobre 2014

Pagination : 41-46

ISBN : 978-2-84174-674-3

ISSN : 2031-4183

Référence électronique

Annette Becker, «Tragédie vs catastrophe », Témoigner. Entre histoire et mémoire [En ligne], 118 | 2014, mis en ligne le 01 octobre 2015, consulté le 23 octobre 2020. URL : http://journals.openedition.org/ temoigner/897 ; DOI : https://doi.org/10.4000/temoigner.897 


\section{Tragédie vs catastrophe}

Entretien avec Annette Becker, historienne de la Grande Guerre et des violences extrêmes du $X X^{e}$ siècle $^{1}$.

Parlons d'abord de vos années de formation. Comment Annette Becker se retrouve-t-elle historienne de la Grande Guerre, avec cette orientation bien spécifique quilla différencie de ses autres collegues?

Annette Becker : En fait, je suis une historienne de la Grande Gur une thèse d'histoirenmé réveils religieux américar XVIII qui ma toujours interesse, ce qui m'a toujours intéressée, ce ne qu'elles soient protestantes ou qu'elles soient protestantes ou lon critquandon l'oncroit qua do crote lon croit quand on croit et, pami jai, poy ains dire, re tait que les mon ains tir de la commande d'un éditeur d'archéc. J'habitais New York, à York, à ce mont voudrat que túcrives un livre vor les que tu écrives un livre J'ét.s monuments aux morts. " Jétais un peu éton je, nais pas

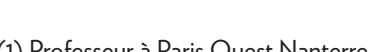

(1) Professeur à Paris Ouest Nanterre
La Défense. HAR EA 4414. IUF Auteur de Biographie de guerre d'Apollinaire (Paris, Tallandier 2009, reeedition textuel 2014) et Belqique occupées (Paris, Fayard, 2010) Voirla Garande Guerre, un autre récit, 1914-20
(Paris, Armand-Colin, 2014).

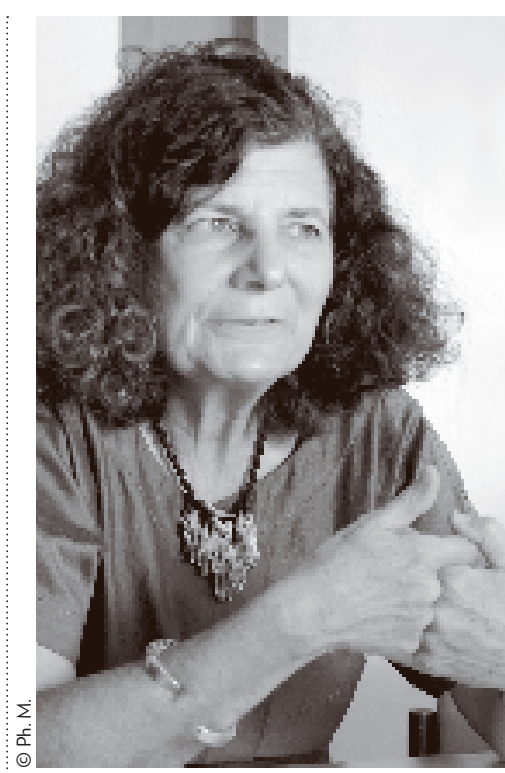

jours eu une passion pour le monuments aux morts et, jusque-

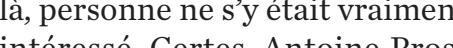
da dans sa these sur les anciens conbatectiven parlait un pen. Il avait port entre l'ant rencien porte moure lan le monument aux morts, mais il social. Il essayait d'y trouver tout, soufla most sije dy trour in ainsi, cest-a-dire lhonnage à la mais il ne voyait pas la façon dont la mort s'était fichée partout. C'est ce que j’ai essayé de mettre en évidence dans ce livre qui a bouleversé ma carrière universitaire, ma vie de chercheuse et ma pensée. Non que je naie jamais travalle sur la violence religieuse

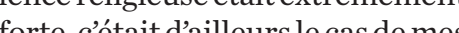
forte, cetait dailleurs le cas de mes pasteurs presbyteriens se battant de façon terible au XVIII siecle.

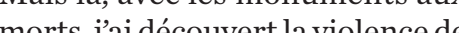
la guerrequétithenautechose évidement Le derien fut mant. Le deuxiène hasare Grès culil a lu mec Jay Winter lorsquil a propód rejoinde léquipe de ce nouvear muséc

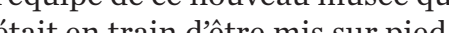
'Historial déremis sur pied: Pourtant dans cettec

Pouns cette équipe, y avait déa quelqu'un qui s'appepère etil père, et il n'etait pas question de été jusqu'aux États-Unis « pour le fuir $\mathrm{Et}$ je le retrouve traville fur me je le retronve travaillant avec ceux qui allaient devenir mer et Gerd Krumeich, liAmícain et l'Allemand. Cela ma cain et isllons

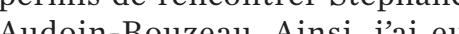
Audoin-Rouzeau. Ainsi, j’ai eu vil chr les monumants auxe tra,a sortie de mon XVIUI où j'étais plus ou moins heurcle. Puis l'Historial, apris quije

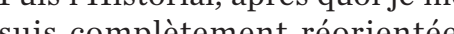

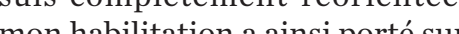
les violin esventesuren

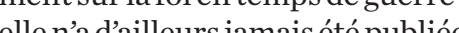

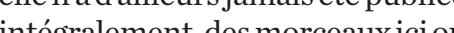
là la Mon prenier veritable live sur foi. Dela mort àla ménorre 


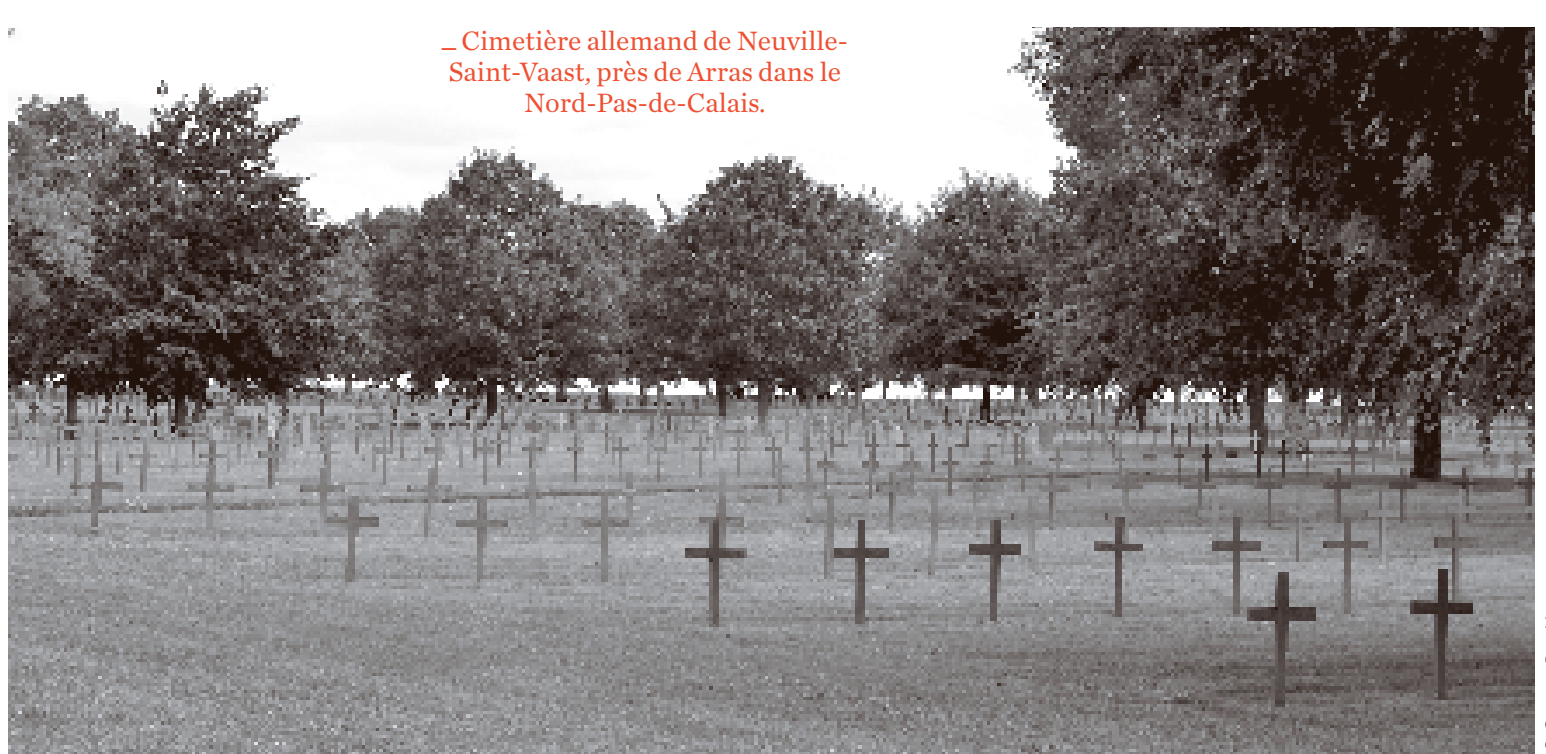

finalement, la foi est un peu passée au second plan, c'est la mort ta mémoire qui ont pris toute la place. Les deux autres intellectuel qui onteu ú ǵtande influence sur moi ont été Étienne Fouilloux et dernier.

Ce travail au sein d'une équipe d'historiens d'une telle envergure a d'historiens d'une
donc été décisif..

Ane décisi...

Annette Becker : Ce que ma apporté ce cercle Première Guerre mondiale, cest une gande ouverture internationale. Ón nepeutpas dialen regardant cincte chos D’où mes difficultés aves Dames. Doù mes dificultés avec certain, est tellemen stellon façon de "faire listoire de la Prepas tellement pardialen, ce n'est pastent

parce qu'ils sont franco-français, prennent la France que par le prisme de la politique fran-

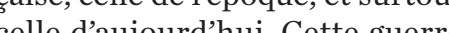
était une guere multipolare, êt une guer inutholaire, ment, d's se dert sur le terito ment, dune part, sur le territoire français et belge et, dautre part sur le territoire gernano-russe et pire ottoman ; elle avait des spepire ottoman, elle avait des spédes belligérants a tous les aspect des belligerants. Ne chercher à a comprendre que d'un seul côté représenta in conprendre. La continutation de cette batalle continue majoritairement à être faite du côte français, comme on ne parvenait pas à se mettre quatre, des cing Il faut aussicomguatre, des chn. Il faut aussiconprendre pourquor les Australien ont traversé le globe pour venir se batrés il n'y quelonnelesyapas derrière chaque Australien!
Franchement, la querelle du Fente de la contrainte est completer le conte cule. Elle prend son origine dans cule. Elle prend son origine dan choses. L’expérience dis nous nouspérience dans laquelle nous hous sommes embarqués il y a une vingtaine d'années quand on a consen à travailler tous manière, ce dont, dune certaine maniere, Retrouver la guerre était la synthese, c'etait cela : essayer drovir con front occidid ent en conaissan mieux Ihistoriographie française, vait se mettre au cour, d'un vait se mettre au coeur d'un phéphénomène que los Angur phènomène que les Anglophones

(2) Annette Becker, La guerre et la foi. De la mort à la mémoire, 1994-1930, Paris, Armand
Colin, «U, histoire contemporaine, „1994. (3) Stéphane Audoin-Rouzeau, Annette
Becker, 14-18, Retrouver la guerre, Paris,
Gallimard, «Bibliotheqque des histoires" Gallimard, «Biblioth
[2000], Folio, 2003.

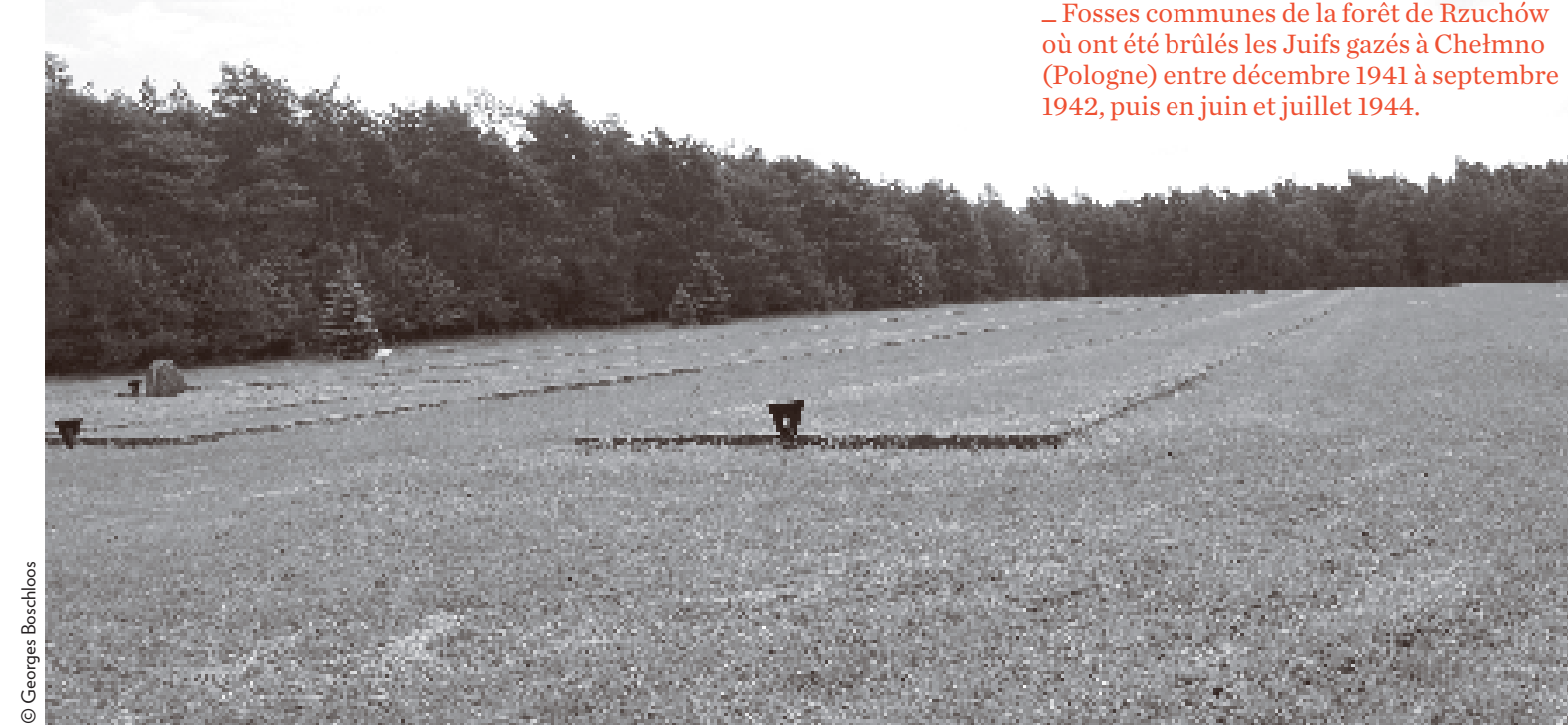

traduit qu'imparfaitement. Il faut arriver à parler de globalité, tout en faisant apparaître les différences nation

En suivant le tracé de l'ensemble de vos publications, on s'aperçoit qu'arrive un moment où vous vous orentez vers les populations civiles.

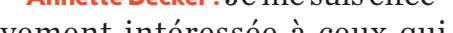
civent niers, les occupés, re ralet, parmi eux, les femmes bien ur. Dunecertaine fa̧on, on savait ch curs. ceux qui conbattaient dune autre façon, me concentrant ainsi sur le Grand Curre Grande Guerre », pour reprendre étaient effectivement oubliés. étaient effectivenent oublies. Cela m'a amenee à travailler sur des détours, je suis arrivée aux
Arméniens, à la spécificité, disons du rejet de l'autre, du racisme e Grande Guerre et a la façon dont on niens m'ont fait aller vers Raphael niens nont de lat ar Lide de là, à la Shoah.

Litre mondiale est un front militaire, fonction les ait pas Il ne sagit pas forctionmerait pas. Il ne s'agit pas seulement du front donestique, fronts qui sont comme des laborafonts qui sont connme des laboracerte en passe de devenir, apres cette guerre, les fronts centraux d'ocerparion diers, fronts de fronts dhopitaux, les dép de massacre, les fronts de ceportations volontas pour nation de civils appelé à partir de 1943, gride. La Gre, a partir de (943, genocide. La Grande Guerre le tou te savoir comituations le tout est de savoir comment elle le recerect conventon peut faire ens avec tout ça.
Nous allons entrer dans une Nourtive. Est-on suffisamment conscient des njeux de celle-ci?

Annette Becker : Ce qui me parât intéressant dans le Centenaire, c'est qu’après avoir mis les «poilus » au premier plan, on s'est aperçu qu’on ne pouvait pas sépala société comme s'ils étaient apnée endessousdeleurpass, dens leurs tranchées. On s'est leurs tranchées. On s'est rend compte que silon ne travaillait pas fronts dociestiques, delin de, lonts domestiques, de larrière, lon ne travallaitpas sur tous ceux différentes pend vies totalenent differentes pendant la Première Gusce mon an

(4) Annette Becker, Oubliés de la Grande
Guerre; Humanitaire et culture de guerre populations occupées, déportés civils, prisonniers de guerre [Noêsis, 1998], édition de poche,
Pluriel/Hachette, 2003. 
Il y a eu là des avancées auxquelles les Belges ont évidemment beaucoup participé. Les territoires occupés, la déportation pour le travail forcé,l'emploi massif de camps de concentration dans l'acception Première Guerre mondiale, c'est-dire des camps d'internement bien différents de ce qu'ils allaien devenir. Néanmoins, prendre en otage des Belges, des Français, de Russes et les transporter comm

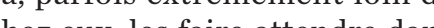
chez eux, les faire attendre dans des miradors cen'estparbelé et Greme l'on ásé hance Guere que lon a été habitué à periner. Ici, je vois tellement de points commans avec la Seconde Guerre mondiale que je peux en cubstier Les saz.

Les civis, ça veut dire tout nonde. Dans le monde entier, je pense à ces Australiens dorgine allenande qui se retrouvent encés pendant quatre ans. On Guerre mo diale pa crenière Guerre mondiale parce qu'ils auraient pu prendre les armes controù certaine quá loccasion du Centea ren encéne compte de lampleur de ce phénomène. Cette prise de conscience est un point positif, début j’avais craint quers qua deu comm le Centenire du pécontine le Centenaire de la d’vavé́e générble vers Une espèce dés etun gérín tés et un recul épistémologique sur Maintenant, je suis un peu plus Meu plus confiante à ce sujet.

On a effectivement tendance à déprécier les commémorations,

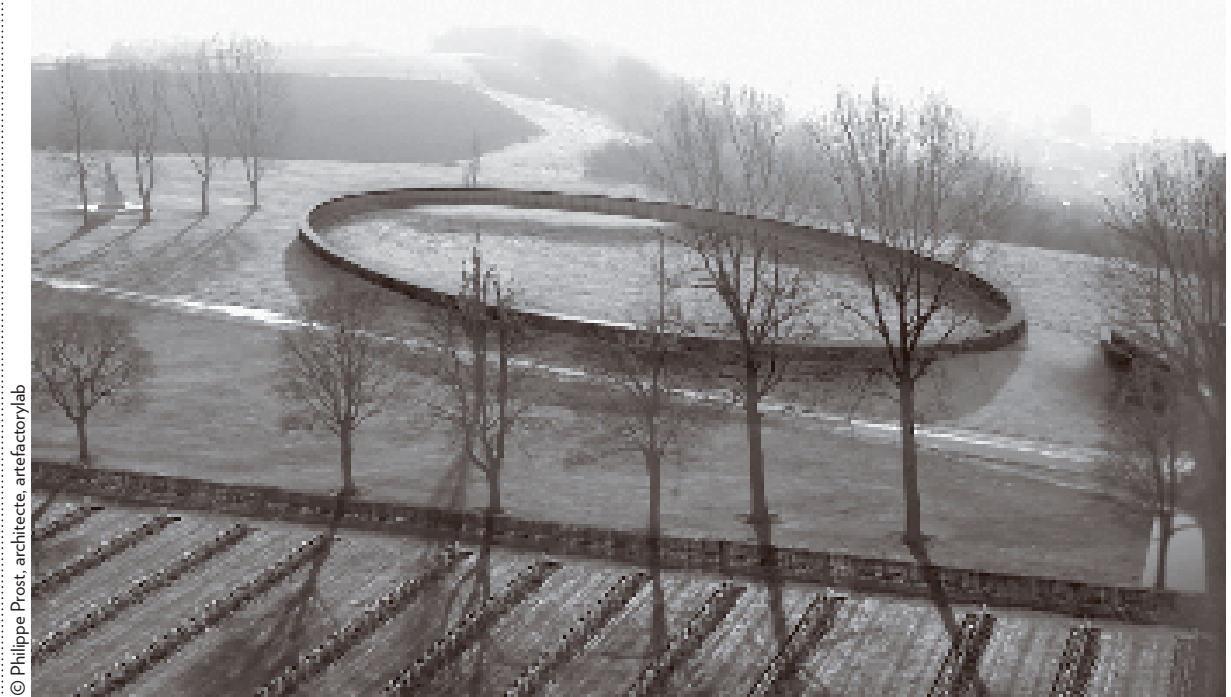
cela signifierait selon vous qu'elles d'unent aussi receler une ouverture ento entre productives de savoir et no sulenente doxa?

crois que l'on A formé des conseils scientifiques pour soccuper du Centenaire festations que ce sont les grandes mávifanta que sont les grandes manifestadu porter. Je pense, par vont lemporter. Je pense, par exemple, a pour le ll novembre : une belle pém le ntration de l'ine belle Cension duconte lans cims lè̀re de Lorette, le long du cinetière de Lorette, un immense les noms de tous les morts Première Guense mots de la Première Guerre mondiale dan la resion du Nord-Pas-de-Calais. cest un toun de le construire,
-Vue d'artiste du futur mémorial de noms de 600000 soldats de toutes les nationalités morts en Flandre française Artois entre 1914 et 1918

trueux. Une entreprise régionale pour parler d'une Guerre monensemble par ordre alphabetique. Ce pour quoi ils se sont battus, le étaient Allemaient très bien qu ils etaient Allemands, Autrichiens, Australiens ou Gallois: tout cela va disparaitre dans cette espèce d'anmort de mass tous frères dans la mort de masse de cette horrible xxe sièc. C'est la gra début du XX été ve 11 novembre 2014 » qui a eté vendue à François Hollande de la région Nord saste-Calas de particulier en particulier par Daniel Percheron, président de la région, ayan tronve des historiens qui se sont consine de justesse de cette diale. Ils mettent les morts tou cela repose sur la vision la plus irénique qui soit: «ils sont tous morts ensemble de la même mort, donc ils avaient la même cause, c'est un gâchis épouvantable.»

Ne faut-il pas voir là une sorte d'assimilation de ces morts-là aux victimes des génocides et, de là, une influence des mémoriaux de la Shoah?

Annette Becker : Absolument, c'est exactement ça qui se passe. Beaucoup d'intellectuels ont mort mortdanslaShoah. Le modèle s'est imposé.

Je dirai que le modèle s'est trouvé, pour des raisons complexes que l'on ne va pas objectiver à l'occasion de cette rancontre, à simposer ou a être imposé, pare a conjonction de discours et de positionnements, d'une façon très spécifique en France.

cker : Cela nie complètement la spécificité de la Première Guerre mondiale. Justement à ce niveau-là, cest un point qui, grâce a to a res, né effet j’ai récens convictions. En Jay Winter pare quil a le mot catastrophe pour laye mière Guere jen'étais pas d'accord. Il faut suel jenctais pas dacolon moí,employer le mot tragedy. Il me rese pas du tout, dans une tragedier, est égaux les uns par rapent est égaux les uns par rapport aux ils étaicts dis etaient égaux dans leur façon que dans une catastrophe comme

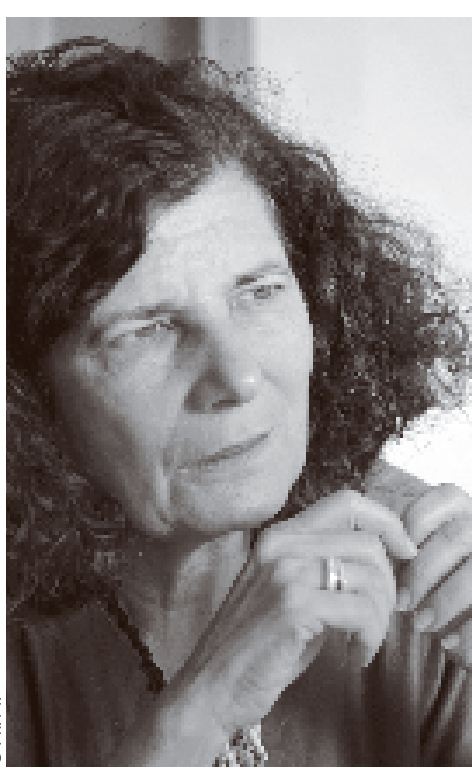
avec la Shoah, il n'y a aucune egasont tués.

Nous, à l'inverse, on se bat pour qu'on ne parle pas de la Shoah comme d'une tragédie.

Annette Becker : Absolument, la Shoah n'est pas une tragédie, c'est catastrophe. Il y a quelque Jay Winter pouvait employer le Jerme de catastrophe ì care l'horreur de la mout de cause de mais la de la nort de masse, massacre Gacre de masse durant b Grande Guerre cesse dirant la Grande Grménienne, ou bia dastrophe triò d'ine ou bien de la deporRusses d'origine allemande) sur le front russe. Hormis ces deux cront russe. Hormis ces deurx moments, la difference est la, et au niveau de la conńchtsevor pour une raison très simple: qu'est-

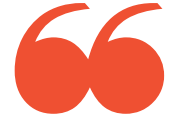

Durant le génocide, avec le massacre de masse : pas de tombes. On fait tout pour effacer les traces.

La catastrophe, elle est là aussi. L'on efface tout signe tangible de la mort.

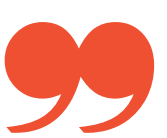

ce qu'on a fait lors de la Première Guerre mondiale? On a essayé partout, sur tous les fronts, dans des d'enterrer les morts. Y compris des corps des ennorts. Y compris es corps des ennemis, pendant guerre, y compris les prisonnier guerre. C'est ce que l'on voit avec guerre. Cest ce que lon voit avec les communes mais morts, dans ancien labibliothè de travail, les écoles, labibliotheque nationale, le musée du Louvre, les gares, etc. La tombe dans les paroisses avec les mout. ments deślises, des synagogur, des temples, etc. On réhumane, des temples, etc. On rénumanise cide, avec le massacre de masse pas de tombes. On fait tous : pas de tombes. On fait tout pour elle est là aussi. Lon affrophe, signe tangible de la mort. Pour signe tangible de la mort. Pour mondiale, sinscrivant en cela à 
la suite d'un phénomène qui a raiment pris forme à partir de la Guerre de Secession, le respect du combattant se prolonge au niveau de la dignite de la tombe que lon létrive de tous les côtés des belligèrants. Dans une catastrophe, n'y a pas de raison, on peut trouver toutes les raisons du monde, mais ens sont toutes nauvaises, toutes fausses, alors on ne respecte pas les morts, on ne leur donne pas de sepulture. Lon essaie de détruire toute trace de la façon de donner la mort, mais aussi tout

Justement, ces historiens, sévertuant à dénoncer la thèse $d u$ consentement, soutenant que tous les soldats ont été poussés à se faire massacrer, malgré eux, se trouven a cautionner ces discours politiques rêniques. Paradoxalement, pour donner une dignite aux victimes, ils qui ô qui ote toute dignité aux victimes et cretrouvent en hagrantecontadiction avec eux-mêmes. Dupes, finalement, de lextension généralisée du modele universalisé du massacre de Asse, du modele génocidaire.

Annette Becker: Absolument. I unisent un modele qui, dans les que durusé toute dignité. Alors que durant la Grande Guerre, cetait linverse.si, aujourdhui, au (an vont dans les cinetières niltaires et en admirent la beaute, en partaires, celle des cimetières militaires allemands extrêmement bien pensés pour se présenter très romantique d'aillours, cela très romantique dailleurs, cela signifie que, pour atroce et indusrestait quelque chose d'un respect

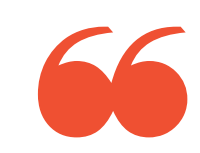

Cette guerre est aussi d'une bizarrerie incroyable, on peut être trois semaines à un endroit sans qu'il

ne se passe rien.

On s'ennuie. Cet aspect est encore peu connu, mal perçu.

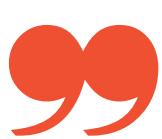

de lêtre humain, ce que l'on n'a pas dans les génocides. Et ça, c'est vraicent a point qui nous se pare ceux qui font une espèce de pacir

Iln'empêche évidemment que cette guerre a été atroce et il ne fautpas fairecroire que, pournous, parler du conkentennent donie la soumrance, Thorreur sans nom de prent a té comnis, ce serait nous non-réco disto non-reflexion historienne sur ce prô̂t août 1914 au 1l novembre 1918 une de bettre, decent de se battre, de recevoir des obus. rerie gur est rerie in à un , semaines à un endroit sans qu' ne se passe rien. On s'ennuie. Cet perçu.
Ill y a cette déviation politique et ce leurre du coté de certin histohens, pour ainsi dire, dupes de leur bon sentiment et de leur crédulité a masse Mas ily a qua nasseres de masse. Mais ily a quand même, ave ductif de savoir, une veritable avaductif de savoir, une verritable avancere scientique, n'est-ce pas?

Annette Becker : Effectivement beaucoup d historiens a travers collectifs ou individuels, beaucoup de travaux voient le jour. coup de travaux voient le jour. On ne parvient plus à tout lire, il y en a trop. Il existe une très sérieuse preparation en anont. D'importâmeque de nes paraissent. De Le problème est que je ne sais pas Le problène est que je ne sais pas ner parce qu'on ne peut pas tout suivre. Il est intérest pas tout que les che che que les chercheurs des pays anglosur les con sur les colonies, sur les peuples françaises Alors que les Franc. resţ̧aises. Alors que les Français restent encore assez en retard làdessus. De plus, un peu partout à à traviors de monde, on profite à travers lé monde, on en profite très bien. Au-delà des fantiques te la tranchée on la tranchée ét des baionnettes, on essaie de reflechir aux conditions de combat, aux occupations, aux massacres de civils, etc. En refficielles ont toutes terations officielles ont toutes tendance à 1918. Je me docaliser sur 19141918. Je me demande bien ce que cela va donner parce quilivay avoir une spece deras-le-bol. Et liyen a encorepour quatre ans. Cest vrai-

Propos recueillis par Luba Jurgenson et
Philippe Mesnard le 5 juin 2014 